\title{
Designing a Diagnostic System of Patients Suspected With Glaucoma Using Retinal Fundus Images
}

\author{
Fateme Moghbeli1 ${ }^{\oplus}$, Mostafa Langarizadeh ${ }^{\star 2} \odot$, Hossein Eghbalian Arani ${ }^{3}$, Azam Orooji ${ }^{1}$
}

1- PhD Candidate of Medical Informatics, Department of Health Information Management, School of Health Management and Information Sciences, Iran University of Medical Sciences, Tehran, Iran.

2- Department of Health Information Management, School of Health Management and Information Sciences, Iran University of Medical Sciences, Tehran, Iran.

3- MSc in Medical Informatics, Department of Health Information Management, School of Health Management and Information Sciences, Iran University of Medical Sciences, Tehran, Iran.

\section{ABSTRACT}

Introduction: Glaucoma is one of eye diseases and is a group of disorders that cause damages to optic nerve. If it does not cure; it can lead to permanent vision loss. There is no sign and pain for this disease and it appears in form of blindness in very advanced phase of its progression. Digital imaging is a useful tool for noninvasive measurements in medical field. By using image processing techniques, many medical images could be processed and analyzed, helping experts to detect diseases, decreasing cost and time of tests and helping to do screening significantly. The main purpose of this study was to suggest an algorithm in order to detect glaucoma suspects in retinal fundus images.

Material and Methods: This study was an original applied study and its algorithm was developed in MATLAB environment using image processing toolbox. It was evaluated by using existed images obtained from RIM-ONE database. In the present paper, Otsu multi-level thresholding algorithm was used along with MICO algorithm to segment the optic cup and disc and estimate their vertical diameter. Results: The algorithm was run on 124 images containing 57 images of glaucoma suspects and 67 images of healthy retina. The sensitivity and specificity were 0.93 and 0.90 respectively.

Conclusion: Suggested algorithm worked fast because light pre-processing has been done. However, there is no system with $100 \%$ correct diagnosis, the results obtained from suggested system was acceptable. Thus using such systems could be helpful for practitioners.
Article History

Received: 2017-09-08

Accepted: 2017-11-30

Published: 2017-12-23

\section{Keywords}

Glaucoma

Image Processing

Optic Cup

Optic Disk

\footnotetext{
* Corresponding Author: M Langarizadeh, School of Health Management and Information Sciences, Iran University of Medical Sciences, Tehran, Iran (Email: langarizadeh.m@iums.ac.ir) 


\section{INTRODUCTION}

Glaucoma is a developing disease of the optic nerve and is known as the second cause of vision loss in the world. It is accompanied with disrupted vision and affects the whole optic system [1, 2] Known as the sneak thief of sight, glaucoma is often diagnosed when it has already developed to a risky state and has damaged one's vision [3]. This disease has irreparable effects on the optic nerve and due to its gradual development, once it is diagnosed it has already damaged the optic nerve tremendously (Fig 1) $[\underline{2}, \underline{4}]$.
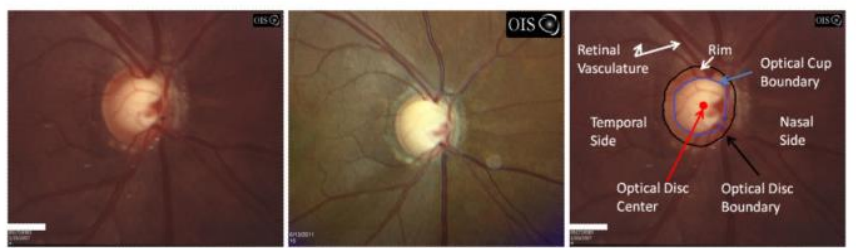

Fig 1: A glaucomatous patient with progressed optic nerve cupping as shown by the color fundus images of optic nerve head (ONH) taken at an early time (left picture) and a late time (middle picture) after about 53 months []].

If diagnosed soon enough, the irreparable damages of this disease can be largely prevented and its development can be delayed or completely controlled [ $2, \underline{3}, \underline{5}-\underline{7}]$. From among its different types, the closed-angle and open-angle only afflicted 60.5 million people worldwide and accounted for the total loss of vision in 8.4 million populations in 2010. The estimated number of its victims until 2020 is 70.6 million. Similarly, the number of total vision loss is estimated to be 11.2 million people [2] . Today, medical imaging is an inevitable part of medical tests and the main affordable means of diagnosing glaucoma and other diseases [ㅁ-10]. In retinal fundus images, blood vessels, optic disc, peripheral ring and optic cup are visible. These can help to estimate several key factors for diagnosing glaucoma such as the cup-to-disc ratio (CDR) as well as the thickness of the peripheral ring of optic disc [11].

There are a number of factors involved in the need for potential image processing techniques in this domain: time-consuming diagnosis of glaucoma parameters in retina image, high workload, lack of efficient expert work force, diagnoses varying across specialists $[\underline{7}, \underline{8}, \underline{11}-\underline{13}]$.

The use of diagnostic software tools save a great deal of time. While in my experience these tools do not identify what the cause of a system problem is, they often provide hints or valuable information about what is in the system and how it is working. Some of these are included with common operating systems at no additional charge, while others are commercial products that range from affordable to rather pricey. Computer-assisted diagnostic software enjoy a high potential for screening plans and help to save large amounts of sources [13]. They help to get rid of the negative consequences of specialists' diverging experiences. In this mode, the software diagnoses whether or not there is one or more symptom of the disease in the image $[\underline{5}, \underline{14}, \underline{15}]$.

In the automated diagnosis of glaucoma, identifying the optic disc and cup is of a key importance. This issue still remains to be a challenge in processing the retinal image. This is due to the differing forms of the cup shape as well as insufficient information on the depth of two-dimensional images ${ }^{16}$. Despite the fact that retinal images contribute greatly to the diagnosis of eye diseases, they are often influenced by disorders and noises $9,17,18$. As a result, they are not much indicative of the details which in turn raises the probability of error in measuring CDR $[\underline{1}, \underline{6}]$. Using automated disease diagnostic methods is time and cost effective since it helps to diagnose patients suspected with the disease and better save doctors' time $[\underline{1}, \underline{6}, \underline{8}, \underline{19}]$.

In their investigation, Zheng et al. made use of a general energy function to apply the global optimization framework via a priority graph cut to determine the shape and location of optic disc and cup. The algorithm introduced in this research was evaluated with non-priority algorithms as well as the commercial optical coherence tomography instruments which outperformed commercial optic coherence tomography instruments []].

In the same year, Cheng et al. conducted a study entitled as "Optic cup segmentation using super pixel classification" to diagnose glaucoma. They made use of 650 images from Singapore Malay Eye Study (SiMES) database in order to teach the software. Subsequently, 1676 images from the same database were tested and analyzed. The overlapping error was used to analyze this algorithm which was estimated as $26 \%$. Moreover, to measure accuracy, the area underneath the Receiver Operating Characteristic (ROC) curve was used which was found to be .811 for the SiMES database and .813 for SCES [20].

In another research entitled as "Efficient reconstruction-based optic up localization for glaucoma screening", Yanwu Xu et al. used a machine learning technique to diagnose the cup in fundus images in order to screen glaucoma. This algorithm was used in ORIGA (-light) as well as SCES databases. To analyze the results, non-overlapping error and the absolute error of CDR were used which were respectively 22.5 and .071 percent. Similarly, the area beneath ROC curve which is used to measure efficiency was found to be .86 . Sensitivity was estimated to be .739 and specificity was $85 \%$ [20].

The present research wants to measure CDR in order to diagnose glaucoma in retinal fundus images which is done via retinal fundus image processing techniques with higher sensitivity and specificity in comparison with other studies.

Diagnostics system monitors application transaction health in traditional, virtualized and cloud environments allowing quick isolation and resolution of problems. It gives providers a common tool to easily collaborate across the entire application lifecycle and release higher-quality applications. Diagnostics software like as we were done in this paper provides deep drill down into transactions from the end user through the back end. And it can serve as a single tool set for pre-production and production to diagnose application performance bottlenecks quickly and improve quality.

The present study aimed to establish a diagnostic system for suspected black blood vessels using retinal fundus images processing. For this study, visible signs in retinal fundus were determined according to scientific texts, and the cup / optic ratio was selected as the most important symptom of the disease that was visible in the retinal fundus. Subsequently, using prototype protocols for image processing and meta programming, the diagnostic system for suspected black-blooded patients was developed based on calculating the cup-to-plate ratio. The proportion of the cup to the optical screen in the images evaluated by the software was calculated by the physician of the surgeon in the neonate and cornea. After the program was executed on the images, the values obtained by the software were compared with the values obtained by the physician.

\section{MATERIAL AND METHODS}

This is a developmental-applied research. Considering the fact that in this software study the design of the automatic determination of the proportion of the cup to the optical plate in 
suspected black-eye patients in two-dimensional retinal images is developed, and since this software can eventually be used to identify suspected cases, therefore, is an applied one.

The present research aims to measure CDR in order to diagnose patients afflicted with glaucoma in retinal fundus images which is done via retinal fundus image processing techniques.

To this aim, the existing images in RIMONE-DB database were used including the retinal fundus images of 169 individuals (Fig 2). From among them, 118 belong to healthy subjects; 12 images belong to the initial stages of the disease; 14 belong to moderate glaucoma; 14 indicate deep glaucoma and 11 represent patients suffering from high pressure in eyes (Fig 2). These images were confirmed by 5 specialists including 4 ophthalmologists and 1 optometrist, and are accessible at http://rimone.isaatc.ull.es [21].

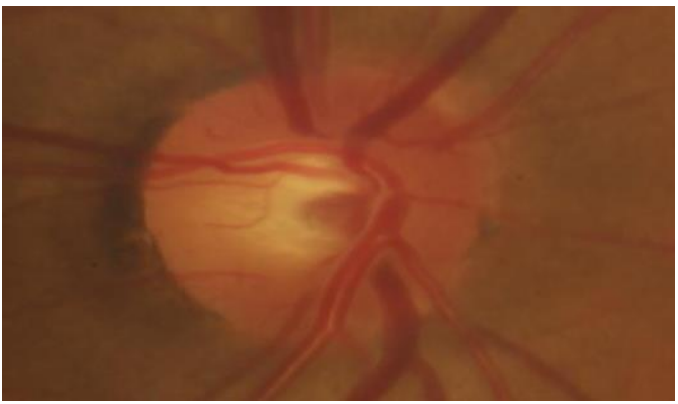

Fig 2: An original retinal fundus images

The algorithm used in this study which estimates CDR without vessel segmentation is comprised of three main levels: a) preprocessing b) main processing c) estimation of CDR and diagnosing the probable affliction with glaucoma. Fig 3 and 4 show the optic cup segmentation and optic disc segmentation by developed algorithm in this research.

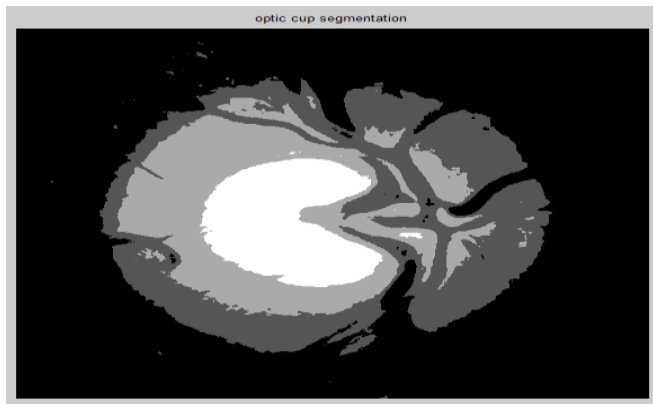

Fig 3: An optic cup segmented by algorithm

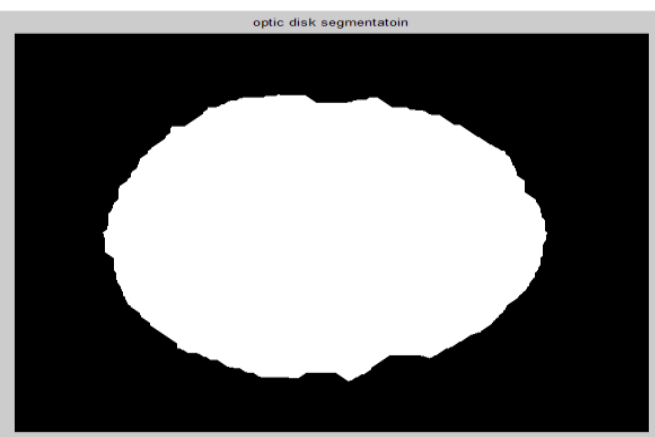

Fig 4: An optic disc segmented by algorithm

\section{Pre-Processing}

In this level, the images are converted from RGB setting to a grayscale image. Only then, ten values are estimated through the multi-level thresholding technique. With the help of these values, the gray-scale image is processed digitally. The outputs of this level are images fit for processing in the next level. This way, the optic disc and cup can be better segmented. Moreover, digital image processing cuts down on the effect of blood vessels as well as the heterogeneous brightness of the background.

\section{Main Processing}

There are two algorithms involved in this level the first of which is for optic disc segmentation. It works based on a recurrent method and the minimization of energy function via a membership function (MF). The input of this algorithm is comprised of images digitized in the pre-processing level. It sets out to segment the optic disc through the segmentation and specification of homogeneous tissues and the values obtained from MF. This MF was already used in a study entitled as "Multiplicative intrinsic component optimization (MICO) for MRI bias field estimation and tissue segmentation" in 2014 to segment tissues [22]

In this algorithm, an initial estimation of an optimal value was made for thresholding via Otsu algorithm and, therefore, a mask was created for the image. Subsequently, according to the algorithm, another mask is created for the image. A multiplication of these two masks yields the final optic disc segmentation. Eventually, to obtain a more homogeneous image without the remaining effects of vessels and noises, an arithmetic morphology was run via the structural element of a circular disc $(\mathrm{r}=30$ pixels) on the processed image. The result was used for the following estimations. The second algorithm which acts based on the multi-level thresholding technique receives the gray-scale images and specifies the optic cup scope. Fig 5 shows the Algorithm flowchart.

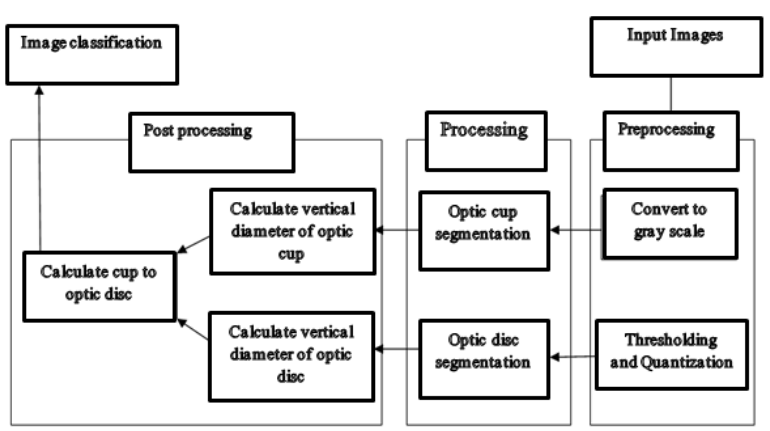

Fig 5: The algorithm trends

\section{Estimations}

Once the segmentation of the optic disc and cup, the result images were used to measure the vertical diameter of the optic disc and cup. To do this, pixels which contained both the expected and specified values in the tagged and segmented images were counted. In order to speed up the counting of vertical diameter pixels, counting extra areas and margins were not taken into account.

First of all, the vertical diameter of the optic cup was calculated. Since the optic cup is located within the disc and they share rather the same diameter, pixels containing the expected values in the vertical diameter of the optic disc were also counted. The diametric values were reported in pixels and a division of these two values yielded CDR. In case, the result was below .4, the image 
would be considered as belonging to a healthy subject. Otherwise, the subject would be considered as suspected with glaucoma.

\section{RESULTS}

The above-mentioned algorithm was run in MATLAB via a number of functions in MALAB tool box. The images used were color or gray and in different sizes. All of which were .bmp type. Once the algorithm was run on these images and the vertical diameters of the cup and disc were estimated, the software provided suggestions based on the CDR and the suspected/healthy subjects. As mentioned previously, if the estimation was below .4, one was diagnosed as healthy. Otherwise, s/he was suspected with the disease.

The results of the algorithm were compared and contrasted with doctor's examination. Running the algorithm on 124 images in the database revealed that 57 belonged to subjects whose CDR was above .4 and were suspected with the disease. 67 images were found to belong to subjects with normal CDR. The sensitivity $($ Sensitivity $=\mathrm{TP} /(\mathrm{TP}+\mathrm{FN})$,$) and specificity ($ Specificity $=\mathrm{TN} /$ $(\mathrm{TN}+\mathrm{FP})$ ) of this algorithm were respectively $93 \%$ and $90 \%$.

Table 1 shows the comparison of this paper with the related ones and it compares their results according to the most important factors like the sensitivity and specificity. Table 1 shows that our algorithm with the new technique of image processing has the best performance according to the other studies.

Table1: Comparison of methods and results in different articles

\begin{tabular}{|c|c|c|c|}
\hline Row & Aims & Results & Reference \\
\hline 1 & $\begin{array}{l}\text { Make use of a } \\
\text { general energy } \\
\text { function to apply } \\
\text { the global } \\
\text { optimization } \\
\text { framework via a } \\
\text { priority graph cut } \\
\text { to determine the } \\
\text { shape and location } \\
\text { of optic disc and } \\
\text { cup by new } \\
\text { developed } \\
\text { algorithm }\end{array}$ & $\begin{array}{lr}\text { New algorithm } \\
\text { has better } \\
\text { performance than } \\
\text { experts' decision }\end{array}$ & [7] \\
\hline 2 & $\begin{array}{l}\text { Diagnose glaucoma } \\
\text { by new algorithm }\end{array}$ & ROC curve: 0.81 & [22] \\
\hline 3 & $\begin{array}{l}\text { Use a machine } \\
\text { learning technique } \\
\text { to diagnose the cup } \\
\text { in fundus images in } \\
\text { order to screen } \\
\text { glaucoma }\end{array}$ & $\begin{array}{l}\text { Sensitivity : .739 } \\
\text { specificity: } 85 \%\end{array}$ & [23] \\
\hline 4 & $\begin{array}{l}\text { measure CDR in } \\
\text { order to diagnose } \\
\text { patients afflicted } \\
\text { with glaucoma in } \\
\text { retinal fundus } \\
\text { images which is } \\
\text { done via retinal } \\
\text { fundus image } \\
\text { processing } \\
\text { techniques }\end{array}$ & $\begin{array}{l}\text { Sensitivity: } 0.93 \\
\text { specificity: } 0.90\end{array}$ & Our study \\
\hline
\end{tabular}

\section{DISCUSSION}

The present research sought to estimate the optic cup to optic disc ratio in the retinal fundus images of patients suspected with glaucoma. It attempted to make use of fast algorithms [19, 23]. Therefore, a two-level algorithm was used. For segmentation and estimation of the vertical diameter of the optic cup and disc, separate algorithms were employed. In case the result was below .4 , the subject would be considered as healthy. Otherwise, s/he would be suspected with glaucoma. This algorithm was run on 124 images located in RIM-ONE database.

From among all these images, 57 images were found to be suspected with the disease and 67 were taken as healthy. A comparison of the estimates with the measurements made by a cornea and glaucoma specialist found the sensitivity and specificity of the system to be $93 \%$ and $90 \%$, respectively. This attests to the proper diagnosis of the algorithm used which was found to outperform other algorithms used in this domain.

Due to the fact that it does not have a time-consuming preprocessing level and requires no segmentation of blood vessels, it enjoys a high speed. A limitation of this study was a lack of national databases of retinal fundus images which enjoy both high quality and necessary information on the disease diagnosis, optic cup and disc scope as well as the blood vessels. We suggest the development of a system which can diagnose the developmental stage of the disease as well as the emergence of other symptoms meanwhile. These can be addressed in the future body of research.

Image processing techniques can be used to analyze and diagnose several diseases and tests. In the present research, Otsu multilevel thresholding algorithm was used along with MICO algorithm in the present study to segment the optic cup and disc and estimate their vertical diameter. The sensitivity and specificity of this algorithm, once run on the images of RIM-ONE database, were found to be $93 \%$ and $90 \%$, respectively.

Our approach incorporates to measure CDR in order to diagnose patients afflicted with glaucoma in retinal fundus images from several existing techniques which are based on shape statistics [7, 22] (e.g. the deformable segmentation and the Active Shape Model). In contrast, our approach supports the prior knowledge in the algorithm directly.

Many researchers have tried to use digital image processing knowledge, using images of retinal fundus with the diagnosis of suspected acne susceptible patients. In this regard, different algorithms and different methods have been used to detect the cap and optical plane, and even the researchers have tried to use other symptoms such as thinning the neural ring around the optical panel or thinning the nerve strands to detect the disease in image processing systems, but there are still many challenges ahead of the disease $[\underline{7}, \underline{22}, \underline{23}]$.

According to the research findings in the first step, it is necessary to create a retina database for people in Iran. This database is intended to include retina images of people with various illnesses that can be used for different purposes. Also, creating an application that can affect the retinal fundus, due to various abnormalities and noise, will automatically optimize its preprocessing operations, which will also increase the accuracy of the system's detection.

\section{CONCLUSION}

Different image processing techniques and machine learning for segmentation and diagnosis of optical photoconductivity to the 
optical screen are useful for the diagnosis of suspected blackheads. In addition to the copy-to-optical ratio, other effective detection factors can also be measured automatically and with the help of a computer individually or in combination in a single application. These include spotting bladder, thinning of the nerve ring and thinning of the nerve layer. Another area that is of interest to medical specialists is the availability of a system to determine the progression of the disease over time. Our future work would include tests on a larger data set and more evaluations on the automatic detection of optic disc center.

\section{REFERENCES}

1. Wang S. Image processing of OCT glaucoma images and information theory analysis. [MSc Thesis] University of Denver. 2009.

2. zur Glaukomerkennung S. Diffusion tensor imaging analysis of the visual pathway with application to glaucoma. [MSc Thesis] Erlangen University. 2012.

3. Liu Y-Y, Ishikawa H, Chen M, Wollstein G, Schuman JS, Rehg JM. Longitudinal modeling of glaucoma progression using 2dimensional continuous-time hidden Markov model. Med Image Comput Comput Assist Interv. 2013; 16(2): 444-451. PMID: 24579171 [PubMed]

4. Zhang Z, Kwoh CK, Liu J, Yin F, Wirawan A, Cheung C, et al. MRMR optimized classification for automatic glaucoma diagnosis. Proceedings of Annual International Conference of the IEEE Engineering in Medicine and Biology Society; 2011.

5. Zhang Z, Srivastava R, Liu H, Chen X, Duan L, Kee Wong DW, et al. A survey on computer aided diagnosis for ocular diseases. BMC Med Inform Decis Mak. 2014; 14: 80-109. PMID: 25175552 DOI: 10.1186/1472-6947-14-80 [PubMed]

6. Haleem MS, Han L, van Hemert J, Li B. Automatic extraction of retinal features from colour retinal images for glaucoma diagnosis: A review. Comput Med Imaging Graph. 2013; 37(7-8): 581-96. PMID: 24139134 DOI: 10.1016/j.compmedimag.2013.09.005 [PubMed]

7. Zheng Y, Stambolian D, O'Brien J, Gee JC. Optic disc and cup segmentation from color fundus photograph using graph cut with priors. Med Image Comput Comput Assist Interv. 2013; 16(2): 75-82. PMID: 24579126 [PubMed]

8. Kauppi T. Eye fundus image analysis for automatic detection of diabetic retinopathy. [PhD Thesis] Lappeenranta University of Technology; 2010.

9. Langarizadeh M, Mahmud R, Ramli A, Napis S, Beikzadeh M, Rahman WWA. Effects of enhancement methods on diagnostic quality of digital mammogram images. Iranian Journal of Cancer Prevention. 2012; 3(1): 36-41.

10. Zarghani H, Bahreyni Toossi MT. Evaluation of organ and effective doses to patients arising from some common X-ray examinations by PCXMC program in Sabzevar, Iran. Iranian Journal of Medical Physics. 2016; 12(4): 284-91.

11. Odstrčilík J. Analysis of retinal image data to support glaucoma diagnosis. [MSc Thesis] University Of Technology, Brno; 2014.

12. Fengshou Y. Extraction of features from fundus images for glaucoma assessment. [MSc Thesis] National University of Singapore; 2011.

13. Safdari R, Kadivar M, Langarizadeh M, Nejad AF, Kermani F. Developing a fuzzy expert system to predict the risk of neonatal death. Acta Inform Med. 2016; 24(1): 34-37. PMID: 27041808 DOI: 10.5455/aim.2016.24.34-37 [PubMed]
14. Karami M, Fatehi M, Torabi M, Langarizadeh M, Rahimi A, Safdari R. Enhance hospital performance from intellectual capital to business intelligence. Radiol Manage. 2013; 35(6): 30-5. PMID: 24475528 [PubMed]

15. Maghsoudi $B$, Langarizadeh $M$, Nilforushan N. Decision support system for age-related macular degeneration. Iranian Journal of Medical Physics. 2017; 14(3): 141-8.

16. Wong DW, Liu J, Tan NM, Fengshou Y, Cheung C, Baskaran M, et al. An ensembling approach for optic cup detection based on spatial heuristic analysis in retinal fundus images. Conf Proc IEEE Eng Med Biol Soc. 2012; 2012: 1426-9. PMID: 23366168 DOI: 10.1109/EMBC.2012.6346207 [PubMed]

17. Bayani A, Shahmoradi L, Langarizadeh M, Radmard A, Nejad A. Quality improvement of liver ultrasound images using fuzzy techniques. Acta Inform Med. 2016 Dec; 24(6): 380-4. PMID: 28077898 DOI: 10.5455/aim.2016.24.380-384 [PubMed]

18. Langarizadeh M, Mahmud R, Ramli A, Napis S, Beikzadeh M, Abdul Rahman W. Effects of image processing techniques on mammographic phantom images: A pilot study. Pertanika Journal of Science and Technology. 2011; 19(1): 67-76.

19. Langarizadeh $M$, Moghbeli F. Applying naive bayesian networks to disease prediction: A systematic review. Acta Inform Med. 2016; 24(5): 364-9. PMID: 28077895 DOI: 10.5455/aim.2016.24.364-369 [ [PubMed]

20. Cheng J, Liu J, Tao D, Yin F, Wong DW, Xu Y, et al. Superpixel classification based optic cup segmentation. Med Image Comput Comput Assist Interv. 2013; 16(3): 421-8. PMID: 24505789 [PubMed]

21. Fumero F, Alayón S, Sanchez J, Sigut J, Gonzalez-Hernandez M. RIM-ONE: An open retinal image database for optic nerve evaluation. Proceeding of 24th International Symposium on Computer-Based Medical Systems (CBMS). 2011.

22. Li C, Gore JC, Davatzikos C. Multiplicative intrinsic component optimization (MICO) for MRI bias field estimation and tissue segmentation. Magn Reson Imaging. 2014; 32(7): 913-23. PMID: 24928302 DOI: 10.1016/j.mri.2014.03.010 [PubMed]

23. Magnetic resonance imaging. 2014;32(7):913-923.

24. Langarizadeh M, Mahmud R. Breast density classification using histogram-based features. Iran J of Med Inform. 2012; 1(1): $1-22$. 